Reprod. Nutr. Dévelop., 1986, 26 (6), 1319-1333.

\title{
Survie embryonnaire précoce de 9 espèces de poissons d'eau douce après un choc de pH appliqué pendant la fécondation ou au cours des premiers stades du développement embryonnaire.
}

\author{
Ch. GILLET, P. ROUBAUD $\left(^{*}\right)$
}

Station d'Hydrobiologie Lacustre, I.N.R.A., 75, Avenue de Corzent, 74203, Thonon, France.

$\left(^{*}\right)$ Equipe de Biologie du Développement, Laboratoire d'Anatomie Comparée, Université Paris VII, 2, Place Jussieu, 75251 Paris Cedex 05, France.

Summary. Prehatching embryo survival of 9 freshwater fish eggs after a $\mathrm{pH}$ shock during fertilization or early stages of development.

The tolerance of freshwater fish eggs to a $\mathrm{pH}$ shock was studied by plunging the eggs for $30 \mathrm{~min}$ into highly buffered acid or alkaline solutions and by measuring the survival rates at the eyed embryo stage. The study was made on eggs from 9 species : common carp, roach, crucian carp, rainbow trout, brown trout, houting, artic char, pike and perch subjected to acid or alkaline shocks either during fertilization or after activation of the egg.

During the life cycle of fresh water fish, the fertilization stage represented a period of high sensitivity to acid shocks for all 9 species : a $\mathrm{pH}$ value of 5 was lethal under our experimental conditions. Cyprinids and rainbow trouts seemed to be the most sensitive to an acid $\mathrm{pH}$. During egg activation, a large increase in the tolerance of the egg to the $\mathrm{pH}$ shock was observed.

\section{Introduction.}

Le pH est l'une des principales caractéristiques physico-chimiques du milieu influençant la survie et le développement des populations de poissons d'eau douce.

L'alcalinisation excessive des eaux n'est pas un phénomène très répandu (Daye et Garside, 1980) même si elle peut apparaître dans des étangs après amendement ou eutrophisation trop importante. Au contraire, l'acidification des lacs et des rivières, sous l'effet notamment des pluies acides, a provoqué la réduction ou la disparition de nombreuses populations de poissons en Europe et en Amérique du Nord (Fromm, 1980 ; Haines, 1981; Howells et al., 1983) : les stades embryonnaires et larvaires paraissent alors être les premiers touchés (Weisbart, 1968 ; Daye, 1980 ; Johansson et al., 1977, 1981 ; Peterson et al., 1980 ; Rombough, 1982). La fécondation, cependant, pourrait être un moment de sensibilité critique (Roubaud et al., 1984 ; Desira et al., 1986) : à ce moment, en effet, pour 
la plupart des poissons d'eau douce, les gamètes sont libres dans le milieu. De plus, l'espace périvitellin qui protégera ultérieurement l'embryon contre les variations de $\mathrm{pH}$, n'est pas encore formé et sa formation même peut être affectée par les bas pH (Peterson et Martin-Robichaud, 1982 ; Peterson, 1984 ; Rombough et Jensen, 1985).

Dans les conditions naturelles, la sensibilité d'une espèce au moment de la fécondation (sensibilité écologique) dépend d'une part de la sensibilité propre des gamètes et de l'œuf en cours d'activation (sensibilité physiologique), et d'autre part, du pouvoir tampon et de la vitesse de dispersion des liquides spermatique et ovarien (ou cœlomique).

Pour connaître l'impact des variations du $\mathrm{pH}$ externe sur la fécondation des poissons d'eau douce il importe donc d'en déterminer la sensibilité physiologique : ceci n'est possible qu'en faisant agir des milieux à pouvoir tampon assez élevé pour lever les effets protecteurs des liquides d'accompagnement des gamètes.

Ainsi avons-nous montré (Roubaud et al., 1984 ; Désira et al., 1986) que la fécondation représente, chez la carpe commune, une période de sensibilité physiologique critique aux $\mathrm{pH}$ acides. Dans ce qui suit nous nous proposons de comparer, face à des chocs acides ou alcalins, la sensibilité physiologique de la fécondation chez la carpe à celle de 8 autres espèces de poissons d'eau douce. De plus, les mêmes chocs de $\mathrm{pH}$ seront appliqués après l'activation de l'œuf pour comparer les sensibilités relatives de la fécondation et du début du développement embryonnaire.

\section{Matériel et méthodes.}

\section{Schéma expérimental.}

Au moment de la fécondation (mise en présence simultanée des gamètes mâles et femelles), ou à différents âges du développement embryonnaire précoce, les œufs sont plongés, pendant 30 min, par lots séparés de 150 à 200 œufs, dans des solutions tamponnées dont le $\mathrm{pH}$ est compris entre 5 et 10 . Après lavage, I'incubation des œufs se poursuit en eau de ville $(\mathrm{pH} \mathrm{7,1;} \mathrm{oxygène} \mathrm{à} \mathrm{saturation} \mathrm{;}$ $\mathrm{CO}_{3} \mathrm{Ca}: 150 \mathrm{mg} / \mathrm{l}$; traces de $\mathrm{NH}_{4}^{+}$; $\mathrm{NO}_{3}^{-}$et phosphates) dans des conditions propices au développement de chaque espèce (température, oxygénation, qualités physico-chimiques de l'eaú régulées et contrôlées) et les pourcentages de mortalité sont déterminés avant l'éclosion. Le critère de discrimination des œufs morts est la coagulation du vitellus (critère des œufs blancs en pisciculture).

Les figures 1 à 11 récapitulent les âges et $\mathrm{pH}$ choisis pour les traitements de chacune des 9 espèces étudiées : carpe commune (Cyprinus carpio L.), gardon (Rutilus rutilus L.), carassin (Carassius auratus L.), truite arc-en-ciel (Salmo gairdneri, Richardson), truite fario (Salmo trutta L.), coregone (Coregonus schinzi palea, Cuvier Valencienne), omble chevalier (Salvelinus alpinus L.), brochet (Esox /ucius L.), perche (Perca fluviatilis L.). 


\section{Préparation des dilueurs expérimentaux.}

Les solutions utilisées pour réaliser les chocs de $\mathrm{pH}$ (dilueurs expérimentaux) ont été préparées suivant Roubaud et al. (1984). Les espèces chimiques destinées à exercer un pouvoir tampon sur le $\mathrm{pH}$ sont utilisées à la concentration de $0,1 \mathrm{M}$ (ou 0,066 M) au voisinage de leur pK d'ionisation (Von Rauen, 1964) : 4-3-aminopropyl morpholine $\left(\mathrm{pK}_{1}=10,2 ; \mathrm{pK}_{2}=6,2\right)$; éthylène diamine $\left(\mathrm{pK}_{1}=9,9\right.$; $\left.\mathrm{pK}_{2}=6,8\right) ;$ glycine $(\mathrm{pK}=9,6)$; tris-hydroxy-méthyl-aminométhane $(\mathrm{pK}=8,2)$; acide acétique ( $\mathrm{pK}=4,75)$.

Le $\mathrm{pH}$ des dilueurs est ajusté par de la soude ou de l'acide chlorhydrique (appareil Metrohm-Herisau E532-Précision : 0,02 Unité pH). L'osmolarité des solutions obtenues est voisine de $150 \mathrm{mosmol} / \mathrm{kg}$ (appareil Vogel 6300).

\section{Obtention des gamètes, fécondation, incubation.}

Cyprinidés : carpe commune, carassin, gardon. - Les gamètes sont obtenus selon le protocole expérimental décrit précédemment (Gillet et Roubaud, 1983) : injection d'extraits hypophysaires de carpe dans la cavité abdominale de géniteurs maintenus en eau froide en fin de vitellogenèse et placés en eau réchauffée $\left(18^{\circ} \mathrm{C}\right.$ pour la carpe et le carassin ; $16^{\circ} \mathrm{C}$ pour le gardon) 8 à 15 jours avant l'injection. Les ovocytes sont fécondés dans les $4 \mathrm{~h}$ qui suivent le début de l'ovulation afin de limiter les processus de vieillissement ovocytaire.

La fécondation et l'incubation sont réalisées, aux mêmes températures respectives, suivant le protocole décrit par Jaoul et Roubaud (1982) :

- dispersion simultanée dans une boîte de Pétri contenant $10 \mathrm{ml}$ de dilueur de fécondation d'environ 200 ovocytes et $20 \mu \mathrm{l}$ de sperme ;

- lavage du sperme à l'eau du robinet. Ce lavage intervient suivant les cas : = à la fin du choc acide, lorsque le choc acide a lieu au moment de la fécondation ; $=$ juste avant le choc, lorsque celui-ci a lieu après 10,20 , ou 30 min d'activation ; = à l'âge de $30 \mathrm{~min}$ dans tous les autres essais.

Trois expériences ont été réalisées avec la carpe, deux avec le carassin et une seule avec le gardon. Chaque expérience utilisait les ovocytes produits par une seule femelle et le sperme mélangé de 3 mâles. Dans certaines séries expérimentales, 3 réplicats ont été préparés pour chacune des valeurs testées du $\mathrm{pH}$ et de l'âge au début du choc (carpe : $1^{\text {er }}$ et $2^{\mathrm{e}}$ essais ; gardon : essai sur la fécondation). Les taux de survie ont été évalués au stade de l'embryon cillé, c'est-à-dire après un temps d'incubation de 60 jours-degrés $C$.

Salmonidés : truite arc-en-ciel, truite fario, corégone, omble chevalier. - Les géniteurs de truite arc-en-ciel, de truite fario et d'omble chevalier proviennent de populations de pisciculture. Les géniteurs de corégones, issus de populations du lac Léman, ont été capturés sur leurs frayères.

Deux séries expérimentales ont été réalisées avec la truite arc-en-ciel, la deuxième en parallèle avec la série sur la truite fario. Deux essais ont été pratiqués avec le corégone (hiver 1984) et un essai avec l'omble chevalier (hiver 1983). Pour obtenir un nombre d'ovocytes suffisant dans chaque série expérimentale, les pon- 
tes de plusieurs femelles ont été mélangées : 10 femelles pour la truite arc-en-ciel, 3 pour la truite fario, 2 pour le corégone, 3 pour l'omble chevalier. Les ovocytes sont récoltés par pression abdominale moins de 8 jours après ovulation spontanée, afin d'éviter l'interférence avec des processus de surmaturation ovocytaire trop importants.

La fécondation a lieu à la température de $10^{\circ} \mathrm{C}$, par lots séparés de 150 à 200 ovocytes : ceux-ci sont dispersés avec $50 \mu \mathrm{l}$ de sperme (obtenu par mélange des spermes de 3 mâles) dans $50 \mathrm{ml}$ de l'un des dilueurs expérimentaux (effets du pH sur la fécondation) ou dans le dilueur d'insémination défini par Billard (1977) (effets du $\mathrm{pH}$ sur le développement). Après 10, 20 ou 30 minutes, selon les cas, les œufs sont lavés et mis à incuber dans une eau courante recyclée, de qualité physicochimique contrôlée à la température de $10^{\circ} \mathrm{C}$ pour les truites, et de $8^{\circ} \mathrm{C}$ pour l'omble chevalier et le corégone.

Les pourcentages de survie sont déterminés à 300 jours-degrés C. pour la truite arc-en-ciel, la truite fario et l'omble chevalier, et seulement à 100 joursdegrés $C$. pour le corégone, en raison des développements de saprolégnales qui perturbent souvent les incubations en clayette pour cette espèce.

Esocidés : le brochet. - Les géniteurs proviennent de l'ésociculture du Paraclet dans la Somme (France). Une série d'essais a été effectuée en 1983 avec les ovules d'une femelle obtenus suivant la technique de Montalembert et al. (1978).

La fécondation et l'incubation ont été réalisées suivant le protocole décrit plus haut pour les Salmonidés. La détermination du pourcentage de survie a été effectuée au stade de l'embryon œillé : à 80 jours-degrés $C$.

Percidés : la perche. - Les géniteurs proviennent d'un petit étang expérimental : les cordons d'ovocytes sont récoltés par pression abdominale après ovulation spontanée : ils sont fragmentés en tronçons de quelques centimètres de long contenant plusieurs centaines d'œufs chacun. Les deux séries d'essais ont été conduites en mars et avril 83. La fécondation et l'incubation se sont déroulées suivant le protocole décrit pour les salmonidés et le pourcentage de survie a été déterminé à la fin de l'épibolie, soit après 45 jours-degrés $C$.

\section{Test statistique.}

Les pourcentages de survie des lots expérimentaux sont comparés à l'aide d'un test de $\chi^{2}$ au risque $5.10^{-3}$ à ceux des lots choisis comme témoins : lots fécondés selon les cas à $\mathrm{pH} 9$ ou 8,2. Les résultats du test sont indiqués sur les figures : une ligne horizontale permet de faire la démarcation entre les points qui ne diffèrent pas (au dessus de la ligne) ou qui diffèrent significativement (au dessous de la ligne) du point représentatif des lots témoins.

FIG. 1 et 2. - Survie embryonnaire de l'ceuf de carpe commune Cyprinus carpio mesurée au stade de l'embryon ceillé $\left(60 \mathrm{j}^{\circ}{ }^{\circ} \mathrm{C}\right)$ et après un choc de $\mathrm{pH}$ appliqué pendant $30 \mathrm{~min}$ au cours de la fécondation, de l'activation ou des tout premiers stades du développement. Fig. 1 : 1er essai ; Fig. 2 : $2^{\mathrm{e}}$ essai ; a : âge au début du choc de $\mathrm{pH}(\mathrm{min})$. Les valeurs situées au-dessous de la ligne en pointillé -..-- diffèrent significativement de la valeur mesurée à $\mathrm{pH} 9,0$ prise comme témoin (test $\chi^{2}$, risque $5.10^{-3}$ ) $; \mathrm{T}$ : demi-écart standard entre 3 répétitions. 

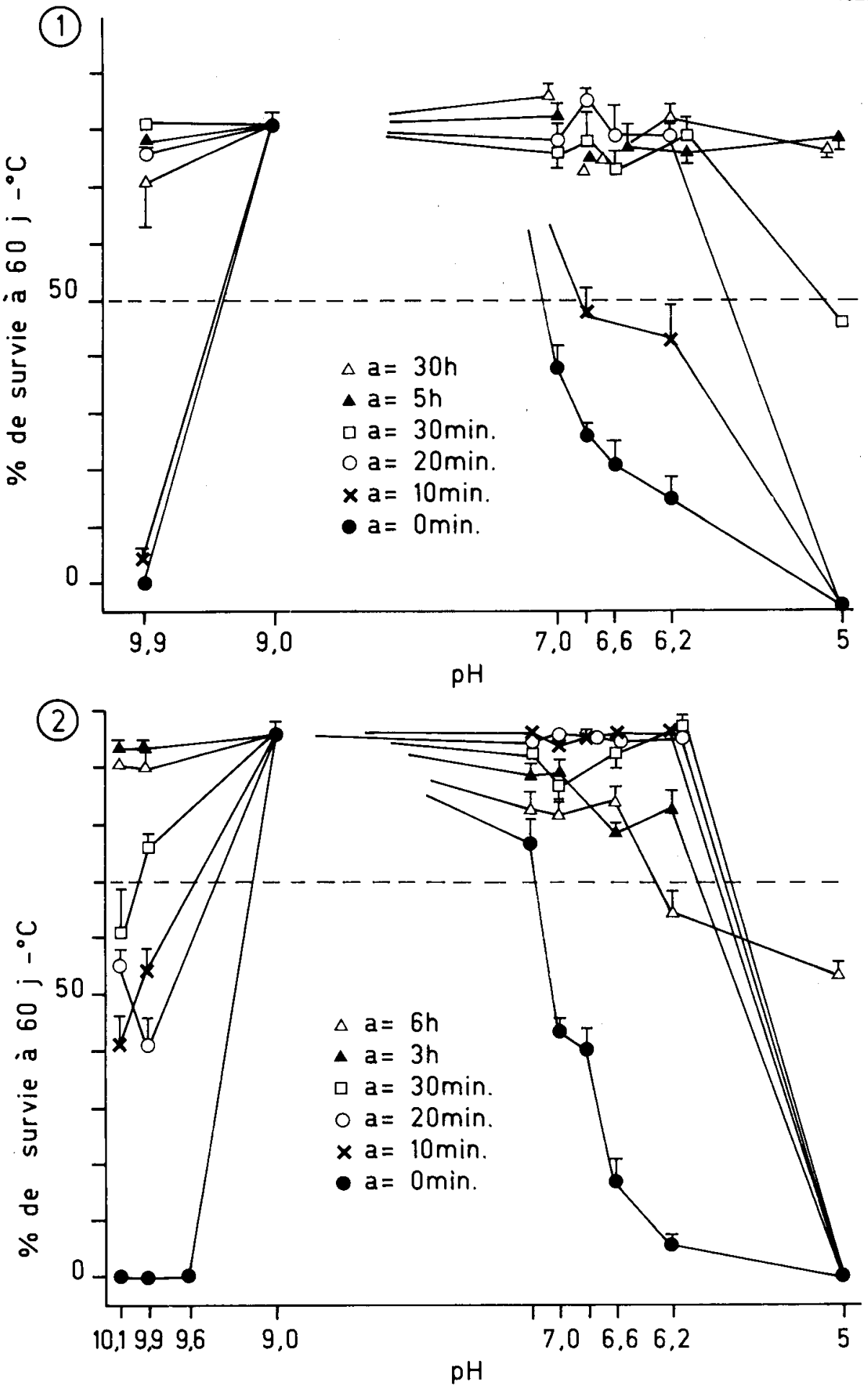


\section{Résultats.}

Les résultats concernant les 9 espèces étudiées sont résumés par les figures 1 à 11 .

La fécondation. - Pour chacune des 9 espèces étudiées, un $\mathrm{pH}$ de 5 s'est avéré, dans nos conditions expérimentales, létal au moment de la fécondation. C'est aussi le cas du $\mathrm{pH} \mathrm{5,4}$ pour l'œuf de corégone (fig. 8) et même du $\mathrm{pH} 6$ pour les œufs de gardon (fig. 3 ) et de brochet (fig. 10). Du côté acide, la borne de l'intervalle de $\mathrm{pH}$ favorable $(\mathrm{I}-\mathrm{pH}-\mathrm{F})$ est voisine du $\mathrm{pH} 7$ pour un premier groupe d'espèces : elle est comprise entre 7 et 7,2 pour la carpe (fig. 1 et 2), entre 7 et 7,5 pour le gardon (fig. 3), entre 6,3 et 6,8 ou 6,6 et 7 pour le carassin (fig. 4) ; de même pour la truite arc-en-ciel, la borne de l'I-pH-F est soit supérieure à 7 (fig. 5) soit comprise entre 6,6 et 6,8 (fig. 6).

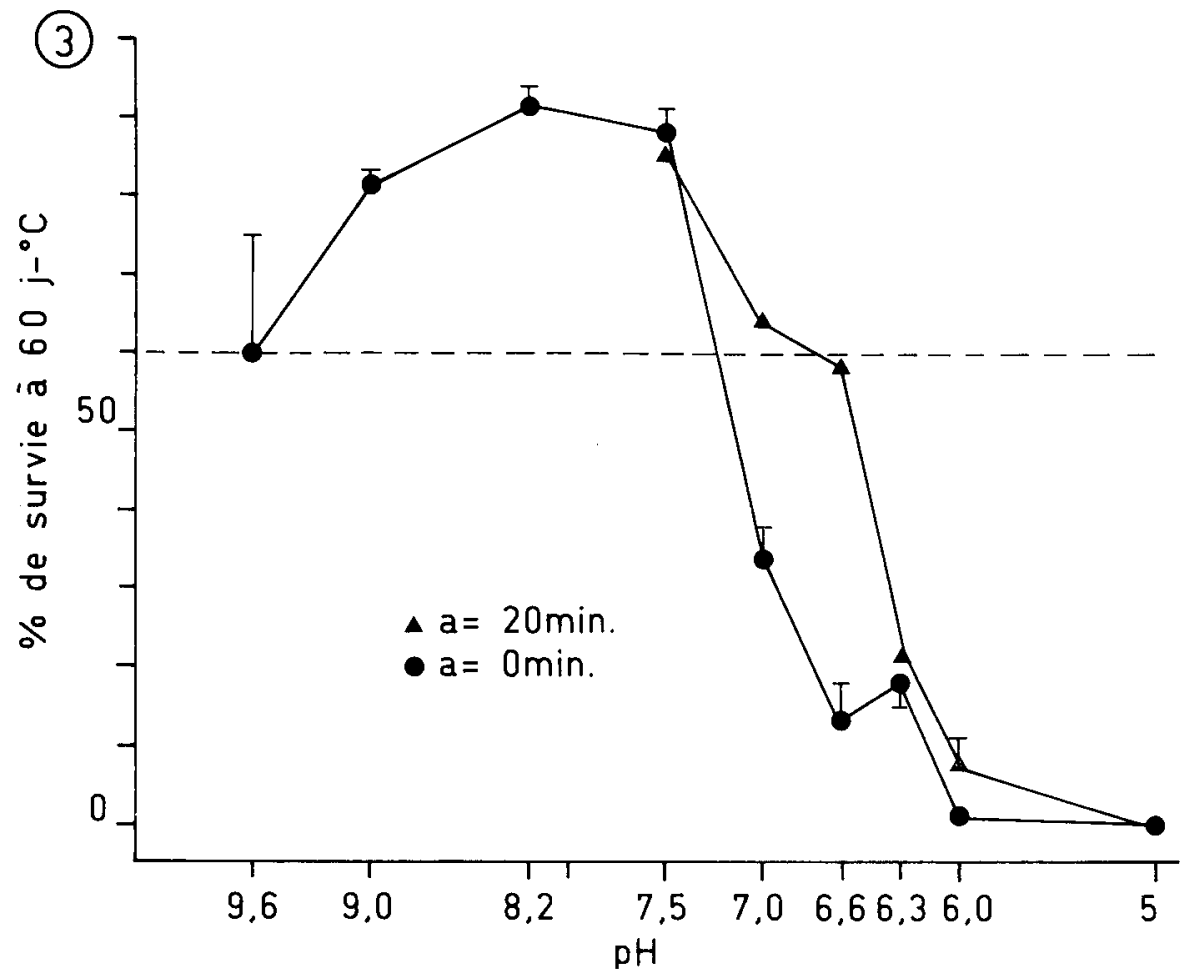

FIG. 3. - Survie embryonnaire de l'œuf de gardon (Rutilus rutilus), mesurée au stade de l'embryon ceille $\left(60 \mathrm{j}^{\circ} \mathrm{C}\right)$ après un choc de $\mathrm{pH}$ appliqué pendant $30 \mathrm{~min}$ au cours de la fécondation ou de la fin de l'activation (explications : cf. fig. 1 et 2).

Pour un deuxième groupe d'espèces, la borne de l'I-pH-F est située au voisinage du $\mathrm{pH} 6$; elle est comprise entre 5 et 6,2 pour la truite fario (fig. 7 ) et l'omble chevalier (fig. 9), entre 5,4 et 6 pour le corégone (fig. 8), entre 5 et 6 pour le brochet (fig. 10), entre 5 et 6 ou 6 et 6,3 pour la perche (fig. 11). 
La baisse de la tolérance lorsque le $\mathrm{pH}$ décroît paraît plus progressive pour le carassin (fig. 4) ou pour la truite arc-en-ciel (fig. 6) que pour les autres espèces.

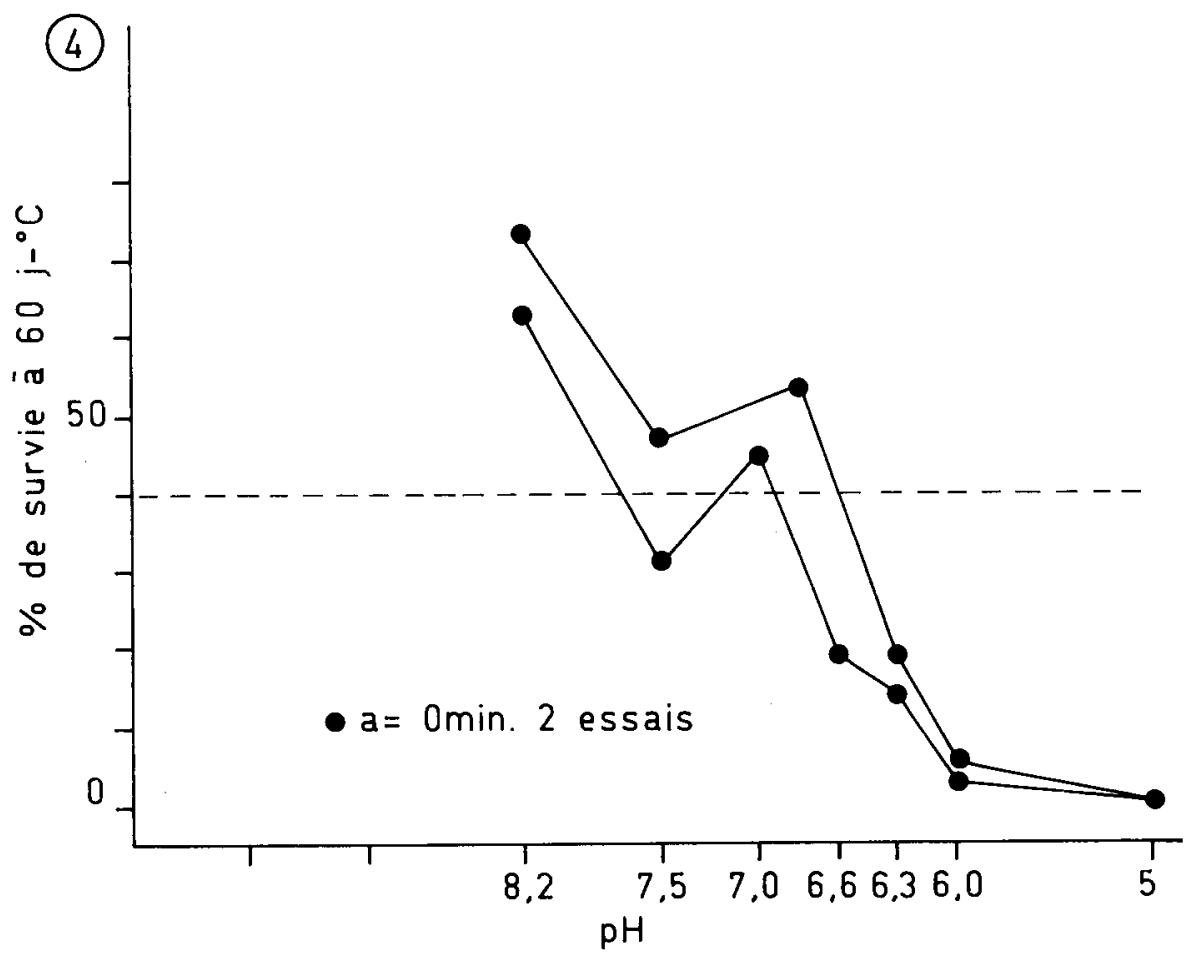

FIG. 4. - Survie embryonnaire de l'œuf de carassin (Carassius auratus) mesurée au stade de l'embryon ceillé $\left(300 \mathrm{j}^{\circ}{ }^{\circ} \mathrm{C}\right)$ après application, au cours de la fécondation, d'un choc de $\mathrm{pH}$ de durée $30 \mathrm{~min}$ (deux essais) (explications : cf. fig. 1 et 2).

Du côté alcalin, les $\mathrm{pH}$ létaux observés sont de 9,9 et 9,6 pour la carpe (fig. 1 et 2 ) et 10 pour la truite arc-en-ciel (fig. 5). Par contre, le $\mathrm{pH} \mathrm{9,6} \mathrm{pour} \mathrm{le} \mathrm{gardon}$ (fig. 3) et pour la perche (fig. 11) ou le $\mathrm{pH} \mathrm{9,5} \mathrm{pour} \mathrm{le} \mathrm{corégone} \mathrm{(fig.} \mathrm{8)} \mathrm{est} \mathrm{encore}$ inclus dans I'I-pH-F.

L'activation. - Pour toutes les espèces où nous l'avons étudiée, l'activation de l'œuf correspond à une augmentation rapide de la tolérance au choc acide. II en est de même pour la tolérance au choc alcalin pour les œufs de carpe ou ceux de truite arc-en-ciel : $30 \mathrm{~min}$ après la fécondation, les embryons sont devenus tolérants aux chocs de $\mathrm{pH}$ extrêmes 6,2 et 9,9 (fig. 1 et 2) ou 6,2 et 10 (fig. 5).

Premiers stades du développement embryonnaire. - Les essais effectués sur des embryons en segmentation (carpe : fig. 1 et 2 ; truite arc-en-ciel : fig. 5 ; truite fario : fig. 7 ; corégone : fig. 8 ; omble chevalier : fig. 9) ou après la fin de l'épibolie (carpe : fig. 1) indiquent un maintien au moins partiel de la tolérance acquise à la fin de l'activation. Cependant, un embryon plus âgé n'est pas nécessairement plus tolérant qu'un embryon plus jeune (fig. 2, 5 et 7). 
(5)
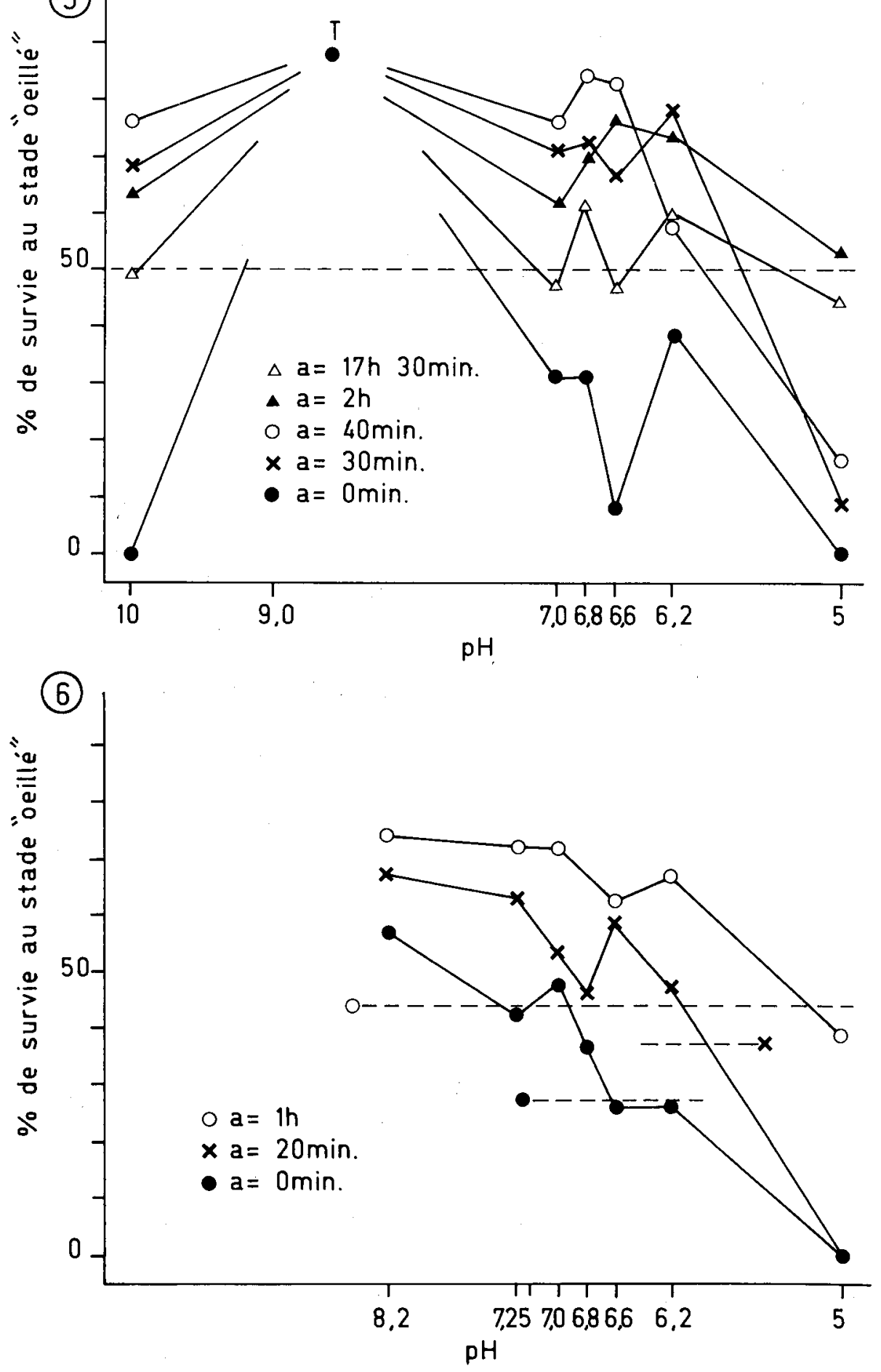


\section{Discussion.}

Les chocs de $\mathrm{pH}$ auxquels nous avons soumis les gamètes et les œufs au cours de la fécondation et des premiers stades du développement embryonnaire font intervenir, à côté du pH proprement dit, d'autres facteurs physico-chimiques tels l'osmolarité, le pouvoir tampon ou la nature des espèces chimiques utilisées comme agent tampon : un examen critique de l'influence que ces divers facteurs peuvent avoir dans nos conditions expérimentales a été présenté antérieurement (Roubaud et al., 1984 ; Désira et al., 1986).

Pour toutes les espèces étudiées apparaît l'exigence, au moment de la fécondation, d'un milieu plutôt alcalin. Cette exigence se manifeste cependant à des degrés variables.

- La sensibilité particulière des cyprinidés aux eaux acides (carpe : Roubaud et al., 1984 et fig. 1 et 2 ; gardon : fig. 3 ; carassin : fig. 4) pourrait être liée au fait que ces espèces se reproduisent à la fin du printemps, dans les eaux chaudes, peu mobiles, et riches en macrophytes (Shikhshabekov, 1974 ; June, 1977 ; Soin, 1977). On sait déjà le rôle majeur joué par la photosynthèse dans la protection des organismes marins contre les pollutions acides (Knutzen, 1981) ; une photosynthèse active pourrait donc déterminer, au contact des œufs de cyprinidés, un milieu nettement alcalin surtout si le comportement de fraie se trouve synchronisé avec des journées chaudes et ensoleillées ;

- Parmi les espèces étudiées autres que les cyprinidés, la truite arc-en-ciel se distingue par sa sensibilité aux eaux acides au moment de la fécondation. Dans des conditions expérimentales différentes Petit et al. (1973) ont montré une baisse importante de la survie embryonnaire lorsque le $\mathrm{pH}$ du milieu de fécondation passe de 9,5 a 7,3. Il se pourrait donc que, pour la truite arc-en-ciel, la courbe de survie en fonction du $\mathrm{pH}$ ne présente pas le maximum en plateau mis en évidence pour les autres espèces (sauf le carassin), mais seulement une valeur optimale située à un $\mathrm{pH}$ très alcalin.

Cependant, l'utilisation d'un dilueur d'insémination d'osmolarité élevée (150 mosmol) et de $\mathrm{pH}$ alcalin pourrait atténuer les effets du vieillissement des ovocytes après ovulation (Petit et al., 1973 ; Billard et Jalabert, 1974). Un effet semblable pourrait d'ailleurs expliquer la décroissance progressive des taux de fécondation observée par Marcel (1981) entre le pH 9 et le pH 7 avec des ovocytes de brochet qui présentaient moins de $50 \%$ de survivants parmi les témoins. Au contraire, dans nos expériences sur cette espèce, la courbe de tolérance présente bien un maximum en plateau entre les $\mathrm{pH} \mathrm{9,6} \mathrm{et} \mathrm{6,3} \mathrm{(fig.} \mathrm{10)} \mathrm{avec} \mathrm{des} \mathrm{taux}$ de survie d'environ $70 \%$.

L'acido-sensibilité élevée de la truite arc-en-ciel au moment de la fécondation est à rapprocher d'observations concernant d'autres périodes du développement :

FIG. 5 et 6. - Survie embryonnaire de l'œeuf de truite arc-en-ciel (Salmo gairdneri) mesurée au stade de l'embryon cillé $\left(300 \mathrm{j}^{\circ} \mathrm{C}\right)$ après un choc de $\mathrm{pH}$ appliqué pendant $30 \mathrm{~min}$ au cours de la fécondation, de l'activation et du début du développement. Fig. $5: 1$ er essai ; Fig. $6: 2^{\circ}$ essai (explications cf. fig. 1 et 2 ). 


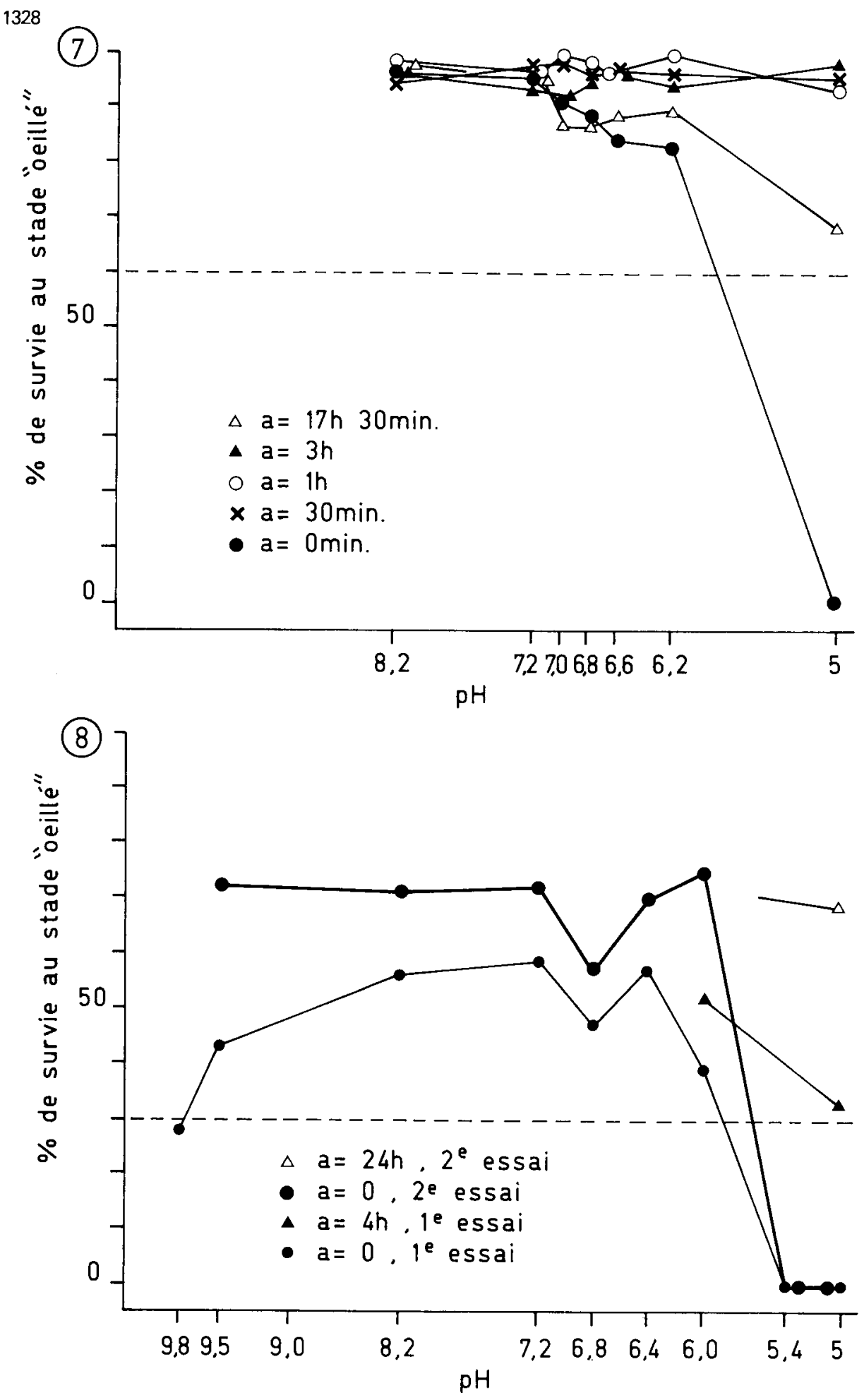


l'embryon de truite arc-en-ciel tolère moins bien que celui de l'omble de fontaine, un traitement par une eau acide après la fécondation (Trojnar, 1977; Kwain, 1975) ; de même, la truite arc-en-ciel est plus sensible que l'omble de fontaine au stade juvénile (Daye et Garside, 1975 ; Kwain, 1975). Il est donc probable que la sensibilité particulière observée pour la truite arc-en-ciel, dans nos expériences, ne tient pas seulement à un vieillissement des ovocytes avant fécondation. Cependant, cette sensibilité pourrait n'être qu'une particularité des souches de pisciculture dont proviennent, en général, les animaux de cette espèce lorsqu'ils sont soumis à l'expérimentation : Brown (1981) a observé que les truites d'origine sauvage sont plus tolérantes que celles qui proviennent d'élevage. Un déterminisme génétique de l'acido-tolérance a déjà été établi, par exemple, pour l'œuf de l'omble chevalier (Gjedrem, 1980) et pour celui de la perchaude (Rahel, 1983). Quant aux souches de pisciculture, elles paraissent avoir, pour la truite arc-enciel, une origine limitée dans l'aire de répartition naturelle de l'espèce (Thorgaard, 1983). Pourtant Daye (1980) n'a pas observé de différence de sensibilité aux eaux acides entre diverses lignées de truite arc-en-ciel ou de saumon atlantique. De plus, la revue de Haines (1981) indique un seuil de disparition des populations naturelles de truite arc-en-ciel nettement plus élevé $(\mathrm{pH} \mathrm{5,5}$ et 6) que celui de huit autres espèces de Salmonidés, ainsi que celui du gardon et de la perche.

- Le niveau élevé de tolérance obtenu dans nos expériences sur l'œuf de perche est peut-être à mettre en relation avec la précocité (stade de fin d'épibolie) de la mesure des taux de survie. On rappellera pourtant que, suivant Muniz et Leivestad (1980), après Johansson et Milbrink (1976) ou Johansson et al. (1977) la perche serait parmi les poissons les plus tolérants au stress acide. D'ailleurs Rask (1983, 1984) trouve, en eau non tamponnée et pour des œufs traités à partir de l'âge de 24 h, c'est-à-dire longtemps après la fin de l'activation, un seuil de tolérance inférieur à pH 5. A l'inverse pourtant, Runn et al. (1977) avancent un seuil de disparition de 5 à 5,5 pour les populations naturelles de la perche, ce qui ne correspond pas à une tolérance particulièrement élevée.

Sensibilité écologique et sensibilité physiologique de la fécondation. - Pour toutes les espèces étudiées, l'activation de l'œuf se traduit par une augmentation importante de la tolérance aux chocs de $\mathrm{pH}$. Ainsi, à rebours, la fécondation apparaît-elle comme une période de grande sensibilité physiologique. Cette observation n'exclut pas la possibilité, comme c'est le cas chez la carpe commune (Désira et al., 1986), que d'autres phases sensibles existent au cours du développement embryonnaire notamment en relation avec le cycle cellulaire des blastomères en segmentation synchrone. Mais, contrairement aux autres périodes du développement précoce, la sensibilité physiologique au moment de la fécondation

FIG. 7. - Survie embryonnaire de l'ceuf de truite fario (Salmo trutta) mesurée au stade de l'embryon ceillé $\left(300 \mathrm{j}^{\circ} \mathrm{C}\right)$ après un choc de $\mathrm{pH}$ appliqué pendant $30 \mathrm{~min}$ au cours de la fécondation, de l'activation et du début du développement (explications : fig. 1 et 2).

FIG. 8. - Survie embryonnaire de l'aeuf de corégone (Coregonus schinzi palea) mesurée au stade du bourgeon caudal $\left(100 \mathrm{j}-{ }^{\circ} \mathrm{C}\right)$ après un choc de $\mathrm{pH}$ appliqué pendant $30 \mathrm{~min}$ au cours de la fécondation, ou du début du développement (2 essais) (explications : cf. fig. 1 et 2 ). 
n'est pas nécessairement déterminante de la sensibilité écologique : en effet, comme nous l'avons mentionné en introduction, les eaux douces naturelles ont un pouvoir tampon réduit si l'on compare à celui des liquides d'accompagnement des gamètes (Plouidy et Billard, 1982). Ceux-ci peuvent éventuellement maintenir autour de l'œuf un $\mathrm{pH}$ favorable, assez longtemps pour que le système protecteur

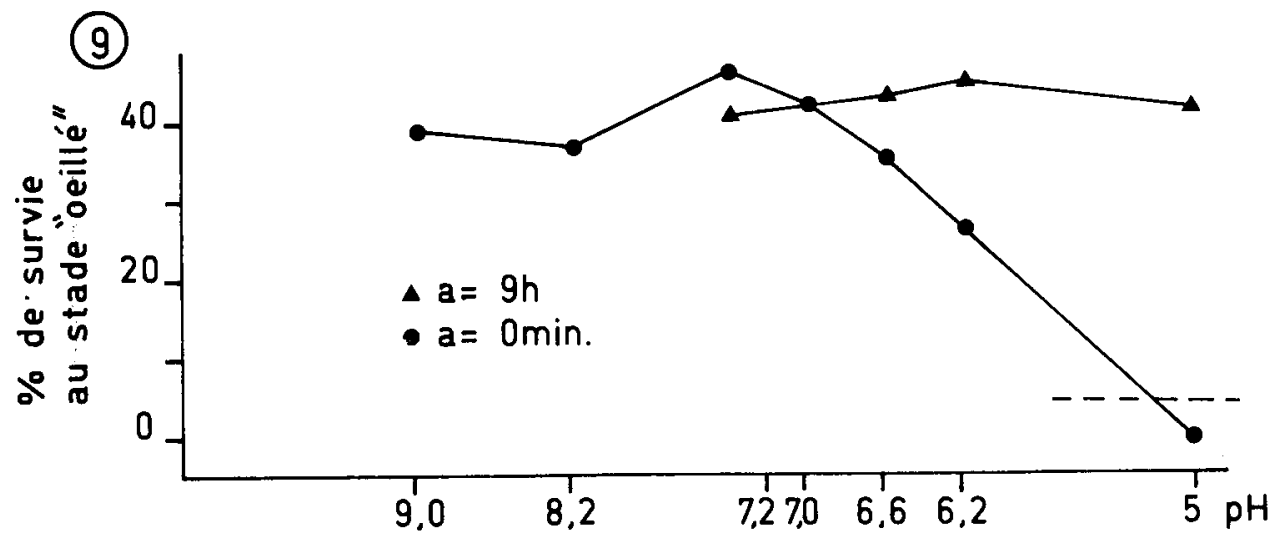

FIG. 9. - Survie embryonnaire de l'œuf de l'omble chevalier (Salvelinus alpinus) mesurée au stade de l'embryon oeille $\left(300 \mathrm{j}^{\circ} \mathrm{C}\right)$ après un choc de $\mathrm{pH}$ appliqué pendant $30 \mathrm{~min}$ au cours de la fécondation ou du tout début du développement (explications : cf. fig. 1 et 2 ).

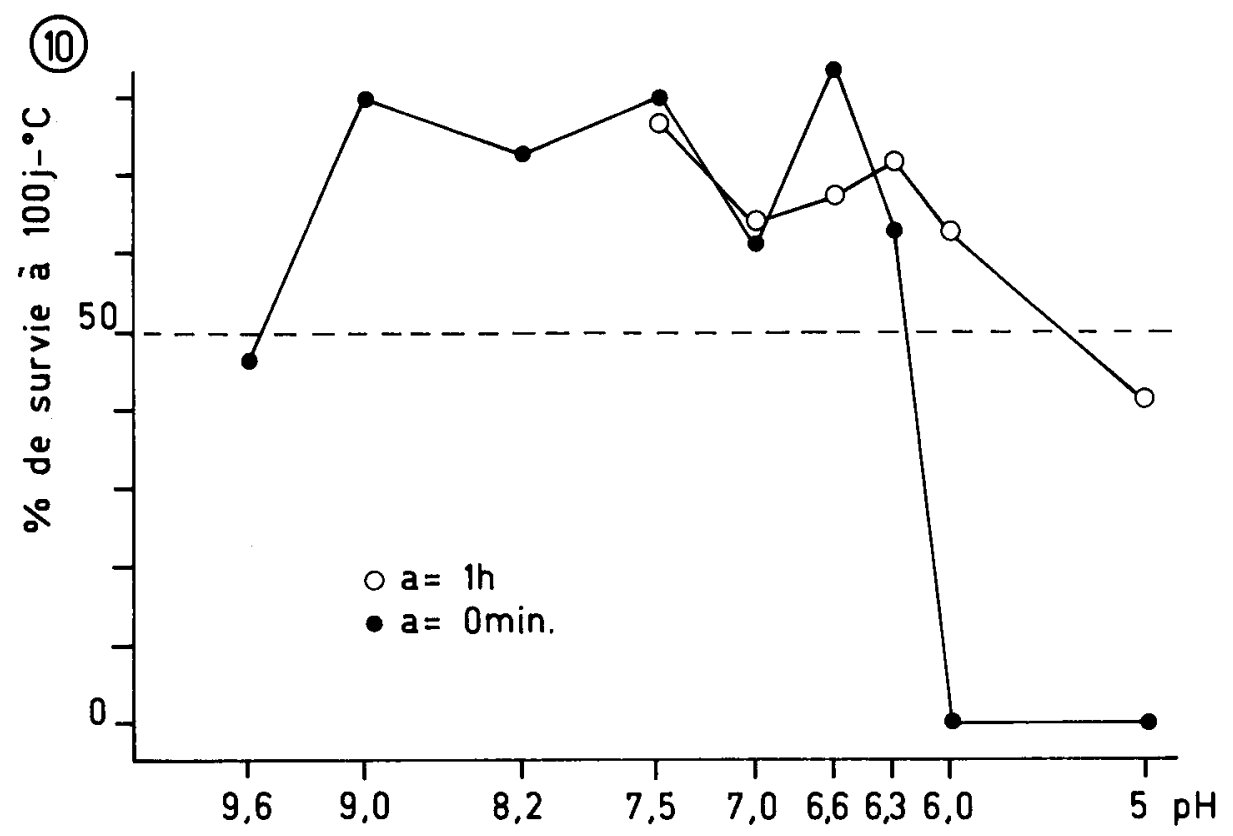

FIG. 10. - Survie embryonnaire de l'œuf de brochet (Esox lucius) mesurée au stade de l'embryon ceillé après un choc de $\mathrm{pH}$ appliqué pendant $30 \mathrm{~min}$ au cours de la fécondation ou de l'activation (explications cf. fig. 1 et 2). 


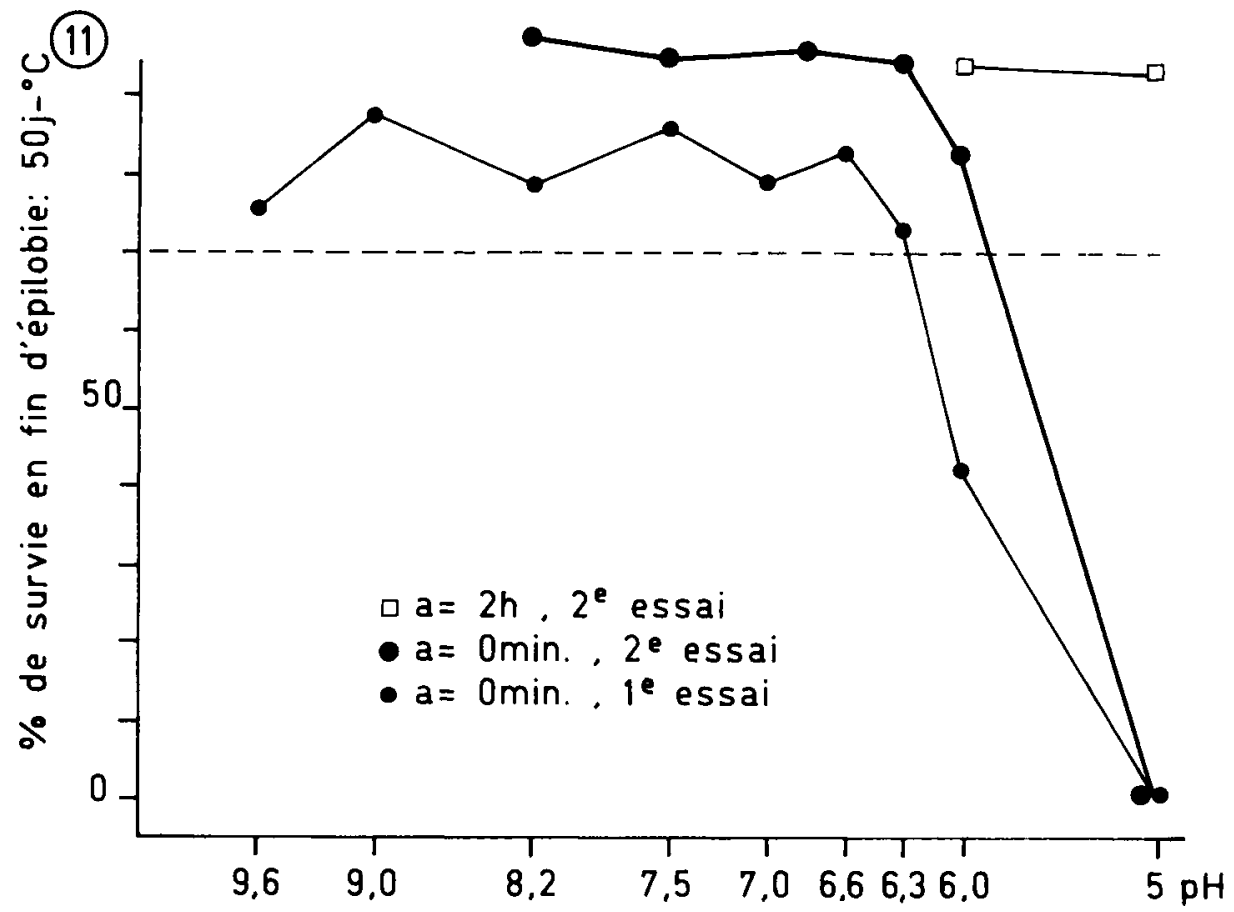

FIG. 11. - Survie embryonnaire de l'œuf de perche (Perca fluviatilis) mesurée à la fin de l'épibolie après un choc de $\mathrm{pH}$ appliqué pendant $30 \mathrm{~min}$ au cours de la fécondation et du début du développement (explications cf. fig. 1 et 2 ).

constitué par la coque et l'espace périvitellin se mette en place. Des études sur la cinétique de la dispersion des gamètes et de leurs liquides d'accompagnement sur les frayères seront donc nécessaires pour décider si la fécondation représente effectivement, dans la nature, une période de sensibilité critique aux eaux acides ou alcalines.

Reçu en juillet 1986. Accepté en septembre 1986.

Remerciements. - Collaboration technique : Installations piscicoles expérimentales : à Jouy-en-Josas : G. Fayemendy, P. Lebrenn, M. Le Villain, D. Marie ; à Thonon-les-Bains : P. Laurent. Bibliographie : C. Chaillou, T. Désira, R. Guyomard. Critique et réalisation du manuscrit : C. Joly.

Ce travail a bénéficié du soutien du secrétariat à l'environnement (Contrat 8340170 ) et du Centre National de la Recherche Scientifique (R.C.P. 080656 ).

\section{Références}

BILLARD R., 1977. Utilisation d'un système Tris-glycocolle pour tamponner le dilueur d'insémination de truite. Bull. Fr. Pisci., 264, 102-112.

BILLARD R., JALABERT B., 1974. L'insémination artificielle de la truite (Salmo gairdneri Richardson). II. Comparaison des effets de différents dilueurs sur la conservation de la fertilité de gamètes avant et après insémination. Ann. Biol. anim. Biochim. Biophys., 14, 601-610. 
BROWN D. J. A., 1981. The effects of various cations on the survival of brown trout Salmo trutta at low pH. J. Fish. Biol., 18, 31-40.

DAYE P. G., 1980. Attempt to acclimate embryo and alevin of Atlantic salmon, Salmo salar, and rainbow trout, Salmo gairdneri, to low pH. Can. J. Fish. aquat. Sci., 37, 1035-1038.

DAYE P. G., GARSIDE E. T., 1975. Lethal levels of $\mathrm{pH}$ for brook trout, Sa/velinus fontinalis (Mitchill). Can. J. Zool., 53, 639-641.

DAYE P. G., GARSIDE E. T., 1980. Development, survival, and structural alterations of embryos and alevins of Atlantic salmon, Salmo salar, continuously exposed to alcaline levels of $\mathrm{pH}$, from fertilization. Can. J. Zool., 58, 369-377.

DÉSIRA T., ROUBAUD P., CHAILLOU C., GILLET C., REINAUD P., 1986. Tolérance physiologique de l'œu de carpe (Cyprinus carpio L.) à un choc acide appliqué au cours de l'activation ou pendant les premières divisions de segmentation. Reprod. Nutr. Dévelop., 26, 393-407.

FROMM P. O., 1980. A review of some physiological and toxicological responses of freswater fish to acid stress. Env. Biol. Fish., 5, 79-93.

GILLET C., ROUBAUD P., 1983. Influence sur la survie jusqu'à l'éclosion des embryons de carpe commune (Cyprinus carpio L.) après traitement, pendant la fécondation et le développement précoce, par le carbendazine, un fongicide antimitotique de synthèse. Water Res., 17, 13431348.

GJEDREM T., 1980. Genetic variation in acid tolerance in brown trout, 308. In D. DRABLOS, A. TOLLAN, Ecological impact of acid precipitation, SNSF project, Proc. Int. Conf. Sandefjord, Norway. Norvegian Inst. for Water Res., Oslo.

HAINES T. A., 1981. Acidic precipitation and its consequences for aquatic systems: a review. Trans. am. Fish. Soc., 110, 669-707.

HOWELLS G. D., BROWN D. J. A., SADLER K., 1983. Effects of acidity calcium and aluminium on fish survival and productivity. A review; J. Sci. Food Agricult., 34, 559-570.

JAOUL A., ROUBAUD P., 1982. Résistance de l'œuf de carpe commune (Cyprinus carpio L., Cyprinidae) à des chocs thermiques chauds ou froids. Can. J. Zool., 60, 3409-3419.

JOHANSSON N., MILBRINK G., 1976. Some effects of acified water on the early development of roach (Rutilus rutilus L.) and perch (Perca fluviatilis). Water Res. Bull., 12, 39-48.

JOHANSSON N., RUNN P., MILBRINK G., 1977. Early development of three salmonid species in acidified water. Zoon, 5, 127-132.

JOHANSSON N., RUNN P., SOHTELL M., 1981. Perivitelline $\mathrm{pH}$ of salmonid eggs in relation to ambiant pH. Water Res. Bull., 17, 994-999.

JUNE F. C., 1977. Reproductive patterns in seventeen species of warm water fishes in a Missouri River Reservoir. Env. Biol. Fish., 2, 285-296.

KNUTZEN J., 1981. Effects of decreased pH on marine organisms. Marin Pollut. Bull., 12, 25-29.

KWAIN W., 1975. Effects of temperature on development and survival of rainbow trout. Salmo gairdneri, in acid waters. J. Fish. Res. Board Can., 32, 493-497.

MARCEL J., 1981. Controle de la reproduction et gestion des gametes de quelques especes de poissons Téléostéens. Diplôme Ecole Prat. Hautes Etudes, 3 sect., Lab. Physiol. Poissons, INRA, Jouy-en-Josas.

MONTALEMBERT G. de, JALABERT B., BILLARD R., 1978. Control of reproduction in northern pike Esox lucius. Am. Fish. Soc. Spec. Publ., 11, 217-225.

MUNIZ I. P., LEIVESTAD H., 1980. Acidification effects on fresh water fish, 84-98. in D. DRABLOS, A. TOLLAN, Ecological impact of acid precipitation, S.N.S.F. project, Proc. Int. Conf. Sandefjord, Norway. Norvegian Inst. for Water Res., Oslo.

PETERSON R. H., DAYE P. G., METCALFE J. L., 1980. The effects of low pH on hatching of Atlantic salmon eggs, 328. In D. DRABLOS, A. TOLLAN, Ecological impact of acide precipitation, S.N.S.F. project, Proc. Int. Conf. Sandefjord, Norway. Norvegian Inst. for Water Res., Oslo.

PETERSON R. H., MARTIN-ROBICHAUD D. J., 1982. Water uptake by Atlantic salmon ova as affected by low pH. Trans. am. Fish. Soc., 111, 772-774.

PETIT J., JALABERT D., CHEVASSUS B., BILLARD R., 1973. L'insémination artificielle de la truite (Salmo gairdneri Richardson). I. Effets du taux de dilution, du $\mathrm{pH}$ et de la pression osmotique du dilueur sur la fécondation. Ann. Hydrobiol., 4, 201-210.

PLOUIDY M. G., BILLARD R., 1982. The chemical composition of the comparison fluids of the gametes in the common carp (Cyprinus carpio). In Reproductive physiology of fish. Proc. Int. 
Symp. Reproductive Physiol of Fish., 1982, Wageningen, The Netherlands. C.J.J. RICHTER et H. J. GOOS ed., Center for Agr. Publ. and Doc., Wageningen.

RAHEL F. J., 1983. Population differences in acid tolerance between yellow perch, Perca flavescens, from naturally acidic and alkaline lakes. Can. J. Zool., 61, 147-152.

RASK M., 1983. The effects of low pH on perch, Perca fluviatilis L. - I. Effects of low pH on the development of eggs of perch. Ann. Zool. Fenn., 20, 73-76.

RASK M., 1984. The effect of low pH on perch, Perca fluviatilis L. - II. The effect of acid stress on different development stages of perch. Ann. Zool. Fenn., 21, 9-13.

ROMBOUGH P. J.. 1982. Effects of low pH on eyed embryos and alevins of Pacific salmon. Can. J. Fish. Aquat. Sci., 40, 1575-1582.

ROMBOUGH P. J., JENSEN J. O. T., 1985. Reduced water uptake and resistance to deformation in acid exposed eggs of steelhead Salmo gaidneri. Trans. am. Fish. Soc., 114, 571-576.

ROUBAUD P., GILLET Ch., BILLARD R., 1984. Influence du pH du milieu au cours de la fécondation sur la survie embryonnaire de la carpe commune Cyprinus carpio. J. Can. Zool., 62, 851861.

RUNN P., JOHANSON N., MILBRINK G., 1977. Some effects of low pH on the hatchability of eggs of perch Perca fluviatilis L. Zoon., 5, 115-125.

SHIKHSHABEKOV M., 1974. Feature of the sexual cycle of some semi-diachromous fishes in the lower reaches of the terek. J. Ichthyol., 14, 79-87.

SOIN S. G., 1977. Some features of the development of the carp (Cyprinus carpio) under hatchery conditions. J. Ichthyol., 17, 759-769.

THORGAARD G. H., 1983. Chromosomal differences among rainbow trout populations. Copeia, 3. $650-662$.

TROJNAR J. R., 1977. Egg hatchability and tolerance of brook trout (Salvelinus fontinalis) fry at low pH. J. Fish Res. Board Can., 34, 574-579.

VON RAUEN H. M., 1964. Biochemisches Taschenbuch. pp. 37-110. Springer-Verlag.

WEISBART M., 1968. Osmotic and ionic regulation in embryo, alevins and fry of the five species of Pacific salmon. Can. J. Zool., 46, 385-397. 